\title{
Recreation of Voltage Fluctuation Using Basic Parameters Measured in the Power Grid
}

\author{
Piotr Kuwalek ${ }^{1} \mathbb{D} \cdot$ Waldemar Jesko $^{2}$
}

Received: 17 October 2019 / Revised: 6 January 2020 / Accepted: 13 January 2020 / Published online: 24 January 2020

(c) The Author(s) 2020

\begin{abstract}
The article presents the preliminary research results on recreating the envelope using the basic parameters measured in the power grid. Six novel algorithms were presented. The quality of proposed algorithms was verified based on the short-term flicker indicator $P_{s t}$ and instantaneous flicker $P_{\text {inst }}$. Probability theory was used in some of the presented algorithms, considering the random operation of real sources of voltage fluctuations. At the beginning of the article an introduction containing the essence of the discussed issues is presented. Next, description of the basic measured parameters in the power grid are presented, being the input data of the tested algorithms. The subsequent part presents the operation of individual algorithms, the correctness of which was verified by laboratory studies and numerical simulations. In addition, the operation of the proposed algorithms for real measurements made for the wastewater pumping station supply circuit is also presented. The metrological interpretation of the results obtained from the numerical simulation and experimental research is discussed, and the conclusions are presented.
\end{abstract}

Keywords Flicker $\cdot$ Power quality $\cdot$ Voltage envelope $\cdot$ Voltage fluctuation indices $\cdot$ Voltage variation

\section{Introduction}

One of the basic types of disturbances that occur in the power grid are voltage fluctuations. This is common problem resulting in incorrect operation of loads supplied from the same circuit as the source of the disturbance. If these loads are lighting sources, then voltage fluctuations can cause the obnoxious flicker affecting the psychophysical state of an observer. Although voltage fluctuations are common and dangerous, they are not clearly consistently defined in the literature. An examples of different definitions of voltage fluctuations are given in [1-6]. For the purposes of the article, voltage fluctuations were assumed as fast changes of the rms values of voltage with the boundary speed equal to $1 \% U_{N} / s$ [7], where $U_{N}$ is the nominal voltage value in the power grid.

Piotr Kuwalek

piotr.1.kuwalek@doctorate.put.poznan.pl

1 Institute of Electrical Engineering and Electronics, Poznan University of Technology, Poznan, Poland

2 Institute of Computing Science, Poznan University of Technology, Poznan, Poland
For practical measurement of voltage fluctuations in the power grid, the following indicators are used [7]:

- Maximum and minimum rms value of voltage $U(t)$,

- Voltage fluctuation indices: the amplitude $\delta U$ and rate $f$ of voltage fluctuations,

- Short-term $P_{s t}$ and long-term $P_{l t}$ indicators of flicker,

- Indicator $\Delta V_{10}$ (eastern countries, e.g., Japan).

However, the existing indicators do not allow: unequivocal identification of the source of disturbance; and the assessment of its character, considering the psychophysical state of the observer of the obnoxious flicker and the operation of loads supplied from the same circuit as the source of the disturbance. The presented problem can be solved by recording the voltage signal and using: wavelet transform [8, 9], Wigner-Ville transform [10], Hilbert transform [11, 12], genetic algorithms [13], or the Kalman Filter [14]. However, at present these methods cannot be used in practical implementations due to the need to store a significant amount of data to implement these algorithms during continuous monitoring of the power grid. In addition, these methods require modification of measuring and recording devices currently used in practice. 
The article presents a proposal to solve this problem by recreating the voltage envelope using basic indicators, which in practice are recorded at power grid measuring points. Assuming that voltage fluctuations can be uniquely identified with amplitude modulation without an attenuated carrier, which is correct for a stiff power grid, recreating the voltage envelope is synonymous with recreating voltage variation. The proposed method does not contribute the recreation of the carrier signal, in which information on voltage distortion (higher harmonics) is stored. However, higher harmonics do not cause voltage variation. Hence, to research on the phenomenon of voltage fluctuations, the proposed method is sufficient.

Six novel algorithms have been proposed to recreation the voltage envelope, considering the random operation of the source of disturbance, which differs them from current literature solutions [15-19]. The proposed approach to recreating voltage variation allows:

- No modification of currently used measuring and recording devices in practice;

- Implementation of the currently proposed signal analysis algorithms [8-13] for the recreated voltage envelope;

- Automatic identification of voltage fluctuation sources in the power grid using the kernel density estimation [20];

- Research on the impact of recreated voltage variation on the state of power loads supplied from the same grid as a disturbing load;

- Verification of connection requirements for new power loads;

- Conversion of voltage fluctuation indices to short-term flicker indicator $P_{s t}$ or $\Delta V_{10}$ indicator [16-18];

- Post-factum assessment of the obnoxious flicker by different light sources, e.g., incandescent light sources or light-emitting diodes [7];

- Obtaining information about the operation of a disturbing load, e.g., about the frequency of changes in the state of the disturbance source [16].

\section{Basic Parameters Describing Voltage Fluctuations}

Basic parameters describing voltage fluctuations are determined for the period of discrimination at the power grid measurement points.

As a standard, the average aggregated rms value of the voltage $U_{\mathrm{AVG}}$, as well as the maximum $U_{\mathrm{MAX}}$ and the minimum $U_{\text {MIN }}$ rms value are determined for the period of discrimination. On the basis of these values, it is possible to pre-classify disturbances and in some cases it is also possible to recreate the voltage envelope [7].
The next parameters, which are measured in power grid are voltage fluctuation indices, i.e., the amplitude $\delta U$ and the rate $f$ of voltage fluctuations. The amplitude of voltage fluctuation $\delta U$ is the maximum or second maximum voltage change $\delta V$ in the period of discrimination. The rate of fluctuation $f$ is the number of voltage change $\delta V$ in the period of discrimination. To increase the diagnostic possibilities of these indices, the rate of fluctuation $f$ in selected $\delta U$ subranges is examined. The increase in subranges allows a more accurate analysis of the phenomenon, however, it also leads to an increase in the memory in which data for the period will be stored. Therefore, in practice, the following $\delta U$ subranges are used: [1.0, 0.9], $(0.9,0.8],(0.8,0.7],(0.7,0.5]$, $(0.5,0.3],(0.3,0.1],(0.1,0)$, which will be later referred to as: $f_{1.0-0.9}, f_{0.9-0.8}, f_{0.8-0.7}, f_{0.7-0.5}, f_{0.5-0.3}, f_{0.3-0.1}, f_{0.1-0.0}[21]$. On the basis of these indices, it is also possible to assess the flicker based on the rate-magnitude characteristics $\delta U=\mathrm{f}(f)$. An exemplary rate-magnitude characteristic $\delta U=\mathrm{f}(f)$ for an incandescent light source is presented in Fig. 1 [15] with a critical curve applied. All points $(f, \delta U)$ above the curve cause the obnoxious flicker. Unfortunately, in the case of these indices, the presented selection of sub-ranges causes loss of information about the features of disturbing loads in the event that in the next recorded time interval a voltage fluctuation source causing significant voltage changes $\delta \mathrm{V}$ appears in relation to the preceding interval.

In most countries of the world, the indicators of shortterm $P_{s t}$ and long-term $P_{l t}$ flicker, to which relevant normative documents refer, are used to assess voltage fluctuations. Flickermeters are used to measure these indicators, which according to [22] are supposed to reflect the processes taking place on the path: the source of light — the eye - the brain of the flicker observer. Thus, these indicators allows assessing only the psychophysical effects of the flicker observer, omitting the features of the disturbing loads and the their impact on other loads in the power grid. Furthermore, the admissible thresholds $P_{s t}$ and $P_{l t}$ were based on a statistical research

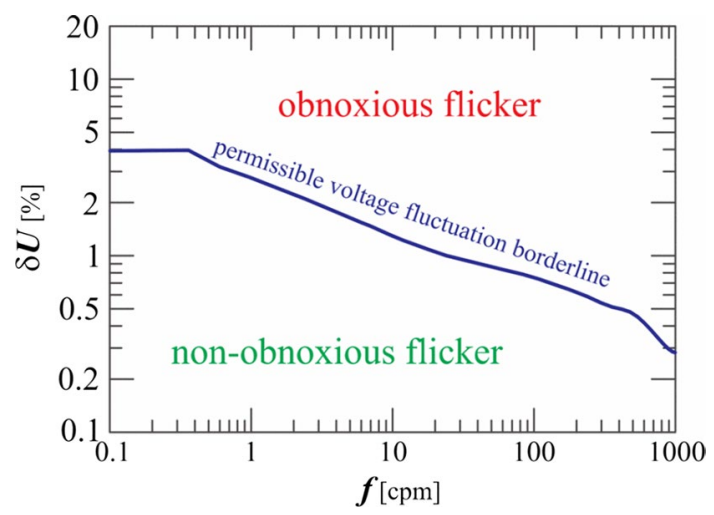

Fig. 1 An exemplary rate-magnitude characteristic $\delta U=\mathrm{f}(f)$ with a fluctuation boundary [15] 
for a $60 \mathrm{~W}$ incandescent light source [23] and inform about the occurrence of negative effects in half of the survey people's, so exceeding the threshold does not necessarily mean that the flicker observer would feel discomfort.

\section{Algorithms for Voltage Fluctuation Recreation}

All the algorithms presented in the article were based on voltage fluctuation indices $(\delta U, f)$, and measures of voltage rms changes: $U_{\mathrm{MIN}}, U_{\mathrm{MAX}}, U_{\mathrm{AVG}}$. Although the voltage fluctuation indices $(\delta U, f)$ give information on the number of occurrences of changes in the rms value and its maximum change in the period of discrimination, they do not provide information about the moment of occurrence of changes, so the voltage fluctuation indices alone do not allow accurate recreation the voltage envelope. Moreover, according to the definition [7], fluctuations do not include slow voltage changes, i.e., the determined voltage fluctuation indices $(\delta U, f)$ include voltage changes with speed above $1 \% U_{N} / s$.

The proposed algorithms have been marked as A1, A2, A3, A4, A5, A6. Input data for individual algorithms, for the studied period of $5 \mathrm{~min}$, are: $U_{\mathrm{MIN}}, U_{\mathrm{MAX}}, U_{\mathrm{AVG}}$, $\delta U=\delta V_{\text {MAX }}, f_{1.0-0.9}, f_{0.9-0.8}, f_{0.8-0.7}, f_{0.7-0.5}, f_{0.5-0.3}, f_{0.3-0.1}$, $f_{0.1-0.0}$. The adopted amplitudes of changes in individual intervals are presented in Table 1.

All algorithms introduce successive voltage changes in such a way that they oscillate around the average $U_{\mathrm{AVG}}$ value and that the sequence of subsequent changes does not exceed the range of $U_{\text {MIN }}$ and $U_{\text {MAX }}$ changes. In turn, differences in the operation of individual algorithms are given below.
(A1) A table has been created in which all changes were accepted in accordance with the assumptions presented in Table 1. Step changes of the rms value (amplitude modulation with a rectangular signal) and even distribution of changes in time were accepted. Then, the change, the index of which in the table is randomly selected in accordance with the uniform distribution, is entered with such a sign that the mean value at the time of change introduction is as close as possible to the measured value $U_{\mathrm{AVG}}$. The only exception is when the change would cause going beyond the scope of [ $\left.U_{\mathrm{MIN}}, U_{\mathrm{MAX}}\right]$, then the change is made so as not to leave the accepted range of changes.

(A2) Two tables were created. In one table there are changes from the interval $(0.1,0.0) \delta U$, considered as "background" (minor fluctuations). In turn, the second table contains the remaining voltage fluctuation. Step changes of the rms value (amplitude modulation with a rectangular signal) and even distribution of changes in time were accepted. Due to the fact that the number of changes $(0.1,0.0) \delta U$ is often much larger than changes from the remaining range, it was assumed that subsequent changes will be introduced in the cycle: one change from the table $[1.0,0.1] \delta U, k$ changes from the Table $(0.1,0.0) \delta U$, where $k$ is the rounding down the number of changes $(0.1,0.0) \delta U$ divided by the number of changes $[1.0,0.1] \delta U$. When $k$ is zero or is undefined, changes are introduced alternately from both tables. In addition, changes are introduced in three phases: in the first phase changes are introduced as positive, in order to reach the nearest $U_{\text {MAX }}$ value, in the second one the changes are introduced

Table 1 List of adopted voltage change values for individual $\delta U$ sub-ranges

\begin{tabular}{lll}
\hline$\delta U$ sub-ranges & $\begin{array}{l}\text { The values of changes relative to } \\
\delta V_{\mathrm{MAX}}\end{array}$ & Comments \\
\hline$[1.0,0.9]$ & 1.0 & - \\
$(0.9,0.8]$ & 0.85 & - \\
$(0.8,0.7]$ & 0.75 & In individual sub-ranges, the number of changes was divided into \\
$(0.7,0.5]$ & 0.69 & three and the amplitudes were taken as extreme and central values \\
& 0.60 & in relation to the sub-compartment. When the number of changes is \\
$(0.5,0.3]$ & 0.50 & indivisible by 3 , then the rest falls on central changes \\
& 0.49 & \\
$(0.3,0.1]$ & 0.40 & \\
& 0.30 & \\
& 0.29 & \\
$(0.1,0.0)$ & 0.20 & \\
& 0.10 & \\
& 0.09 & \\
& 0.01 &
\end{tabular}


as negative to reach the nearest $U_{\mathrm{MIN}}$ value, and in the last phase changes are introduced in the same way as in A1. In contrast to A1, the changes are not randomly selected, but are selected (by searching the table) in such a way that the assumptions of each phase are met (comparing successively introduced changes in voltage with the absolute value of the $U_{\text {MAX }}$ difference and the final value of the reconstructed envelope and with the analogous absolute value in relation to $U_{\text {MIN }}$ ).

(A3) Voltage changes are introduced analogously to A1. However, trapezoidal changes in the rms value of voltage have been assumed, and the introduced changes take place with different time steps (see Fig. 2) using the first-degree Lagrange polynomial interpolation. The time step $d t_{l(\mathrm{i})}$ is determined assuming a constant speed of voltage changes $S R=300 \% U_{N} / s$, where $U_{N}$ is the nominal voltage value in the power grid. In turn, the time step $d t_{2}$ is determined as the difference between the period of discrimination $(5 \mathrm{~min})$ and the sum of time steps $d t_{1(i)}$ for all registered changes, divided by the number of changes.

(A4) The introduction of voltage changes takes place as in A2. Trapezoid voltage changes and a variable time step were assumed in the same way as in A3.

(A5) The introduction of voltage changes takes place as in A1. Trapezoid voltage changes and a variable time step were assumed in the same way as in A3, with the difference that $d t_{1(i)}$ is determined based on the variable speed of voltage changes $S R$. Different values of the rate of change in voltage are randomly selected from the gamma distribution with a shape parameter equal to $300 / 0.7$ and a scale parameter equal to 0.7 . The distribution was chosen because of the support, explicit equation of the mean, mode and variance, and the shape of the distribution.

(A6) The introduction of voltage changes takes place as in A2. Trapezoid voltage changes and a variable time step were assumed in the same way as in A5.

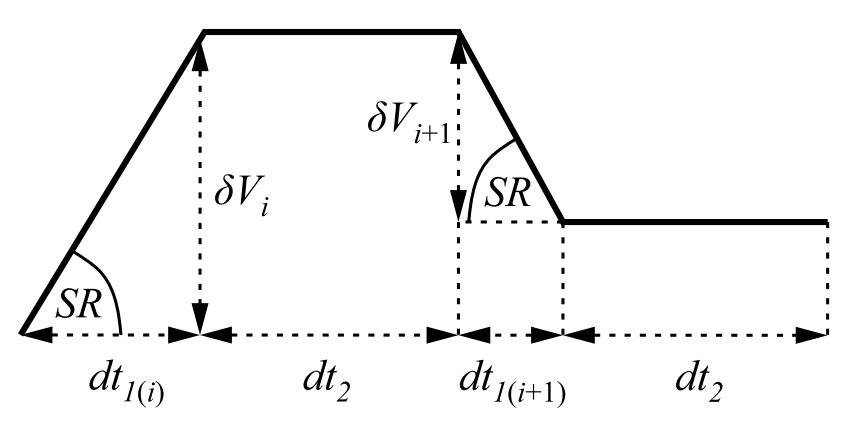

Fig. 2 An example of a fragment of the envelope obtained using the algorithm A3
In addition, the quality of voltage variation recreation by the proposed algorithms was compared with actually the best literature solution [15-19], which has been marked as AR [15]. Description of the algorithm AR operation is given below.

AR The algorithm use the fluctuations alternately when it comes to amplitude: first all of the fluctuations of $f_{0.1-0.0}$ rate are used, then the fluctuations of $f_{1.0-0.9}$ rate, and subsequently $f_{0.3-0.1}, f_{0.9-0.8}, f_{0.5-0.3}, f_{0.8-0.7}$ and $f_{0.7-0.5}$ in that order. The values of voltage changes were adopted as the upper limits of individual sub-ranges. It has been assumed that the rms value should oscillate around the rated value of voltage. For this purpose, the subsequent changes of voltage are introduced in a way which directs the resulting rms value of modulated signal to the rated value - if it is currently greater than the rated rms value, the next change will be introduced with minus sign; if its smaller than the rated value, the next change will be introduced with plus sign. This algorithm introduces subsequent changes evenly in the whole registration period-despite the amplitude the subsequent changes of voltage are added with the same time span. The span is the result of division of total number of seconds in registration period by the total number of fluctuations detected within the registration period [15].

\section{The Exemplary Results of Voltage Variation Recreation}

The same criteria as in [15] were selected to assess the quality of algorithm operation. As test signals were selected deterministic sinusoidal signals (carrier signal) with rectangular amplitude modulation (without an attenuated carrier), which are described by equation:

$u(t)=u_{c}(t) \cdot\left[1+u_{\bmod }(t)\right]$

where $u_{c}(t)$ is the carrier signal described by equation:

$u_{c}(t)=\sqrt{2} U \sin \left(2 \pi f_{c} t\right)$

and $u_{\bmod }(t)$ is the modulating signal described by equation:

$u_{\bmod }(t)=\frac{1}{2} \frac{\Delta U}{U} \frac{1}{100} \operatorname{sgn}\left[\sin \left(2 \pi f_{m} t\right)\right]$.

Based on Eqs. (1)-(3), the modulation depth $(\Delta U / U)$ is determined by equation:

$\frac{\Delta U}{U}=\left(\max \left[u_{\bmod }(t)\right]-\min \left[u_{\bmod }(t)\right]\right) \cdot 100 \%$. 


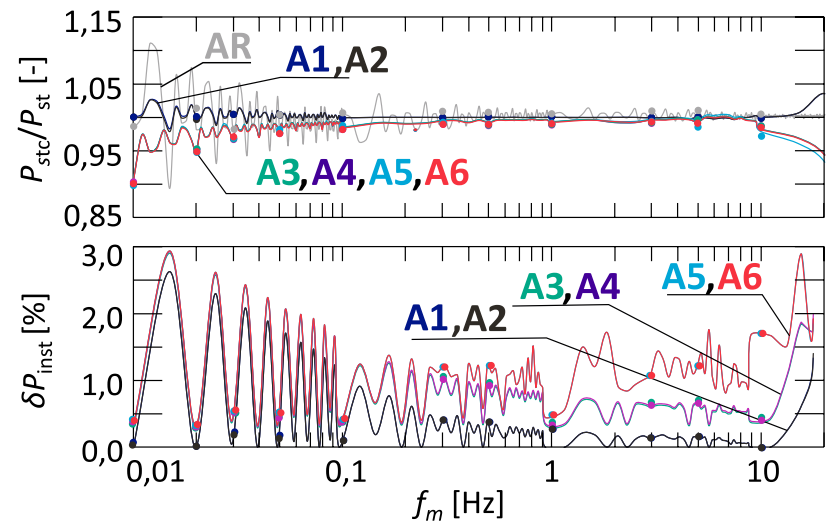

Fig. $3 P_{\text {stc }} / P_{\text {st }}=\mathrm{f}\left(f_{m}\right)$ (top) and $\delta P_{\text {inst }}=\mathrm{f}\left(f_{m}\right)$ (bottom) characteristic for $(\Delta U / U)=0.827 \%=$ const

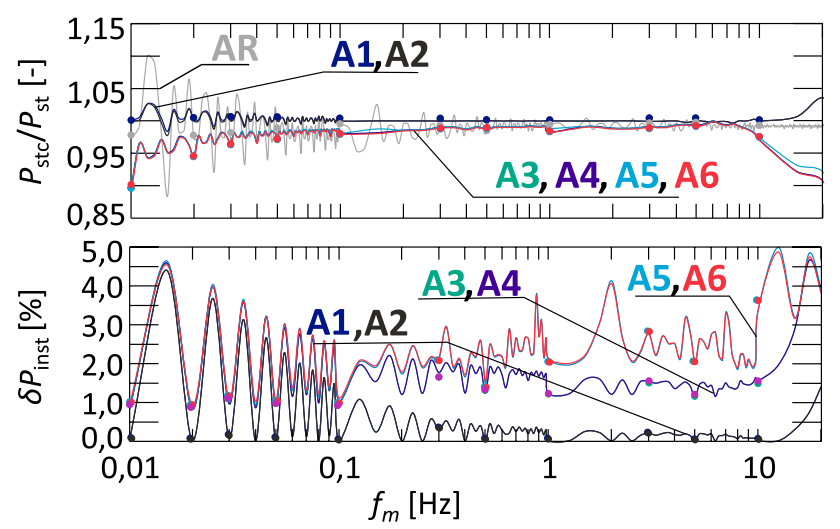

Fig. $4 P_{\text {stc }} / P_{\text {st }}=\mathrm{f}\left(f_{m}\right)$ (top) and $\delta P_{\text {inst }}=\mathrm{f}\left(f_{m}\right)$ (bottom) characteristic for $(\Delta U / U)=1.405 \%=$ const

In the real power grid, the voltage may be distorted, so the carrier signal can be non-sinusoidal. However, in this case, higher harmonics are occurred that do not cause voltage variation. Also, the modulating signal can be non-rectangular signal. However, the rectangular modulating signal causes the most "obnoxious" flicker [24], so it was chosen for research (as in [15]).

For the generated signals, the basic indicators presented in Sect. 2 were measured. The $P_{s t}$ indicator and the instantaneous flicker $P_{\text {inst }}$ were selected as the reference value, which allow assessing the correctness of the operation of individual algorithms. In the research were adopted the same test series as in [15], allowing comparison the presented algorithms with existing literature solutions. Thus; the first measurement series was created using amplitude modulation with constant modulation depth, i.e., $(\Delta U / U)$ it was equal to: $0.827 \%$ (Fig. 3), 1.405\% (Fig. 4), $2.756 \%$ (Fig. 5), $8 \%$ (Fig. 6). In the second series, the modulation depth was changed, so that for each modulation frequency $f_{m}$ the constant $P_{s t}$ indicator was obtained, which was equal to: 0.8 (Fig. 7); 1.2 (Fig. 8); 3 (Fig. 9); 5 (Fig. 10). In the third measurement series, the

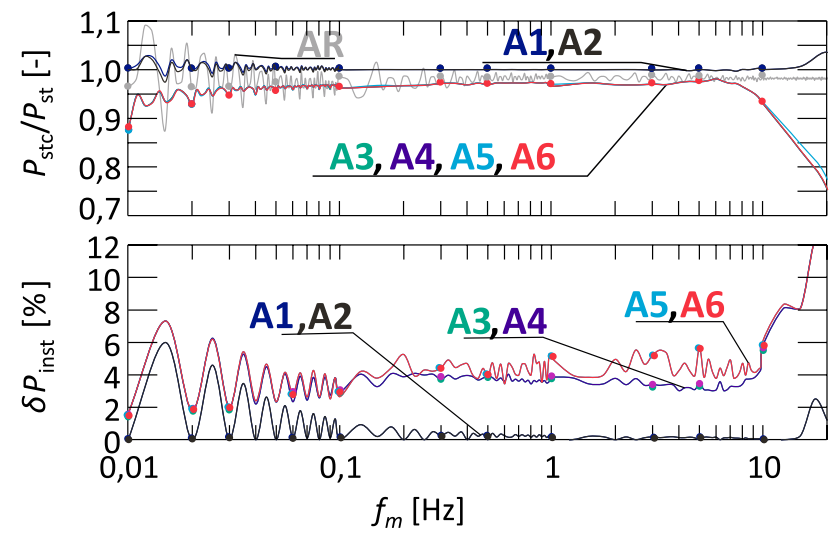

Fig. $5 P_{\text {stc }} / P_{\text {st }}=\mathrm{f}\left(f_{m}\right)$ (top) and $\delta P_{\text {inst }}=\mathrm{f}\left(f_{m}\right)$ (bottom) characteristic for $(\Delta U / U)=2.756 \%=$ const

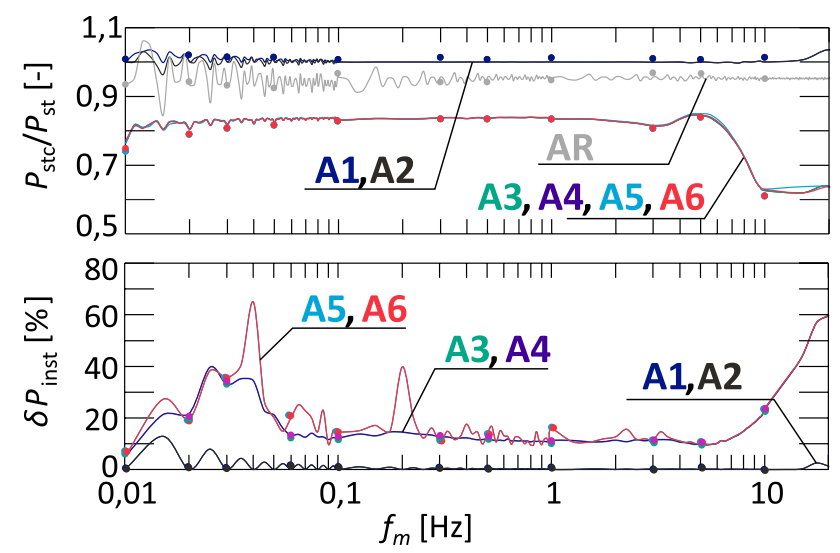

Fig. $6 P_{\text {stc }} / P_{\text {st }}=\mathrm{f}\left(f_{m}\right)$ (top) and $\delta P_{\text {inst }}=\mathrm{f}\left(f_{m}\right)$ (bottom) characteristic for $(\Delta U / U)=8 \%=$ const

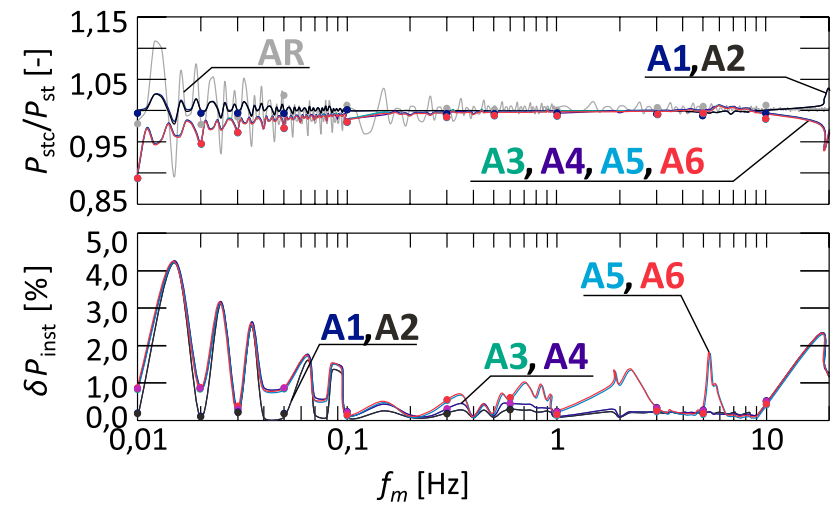

Fig. $7 P_{\text {stc }} / P_{\text {st }=}=\mathrm{f}\left(f_{m}\right)$ (top) and $\delta P_{\text {inst }}=\mathrm{f}\left(f_{m}\right)$ (bottom) characteristic for $P_{\mathrm{st}}=0.8=$ const

modulation depth was changed while maintaining a constant value of $f_{m}$ equal to $0.2 \mathrm{~Hz}$ (Fig. 11), $10 \mathrm{~Hz}$ (Fig. 12), $20 \mathrm{~Hz}$ (Fig. 13). The analysed frequency of modulating signal $f_{m}$ 


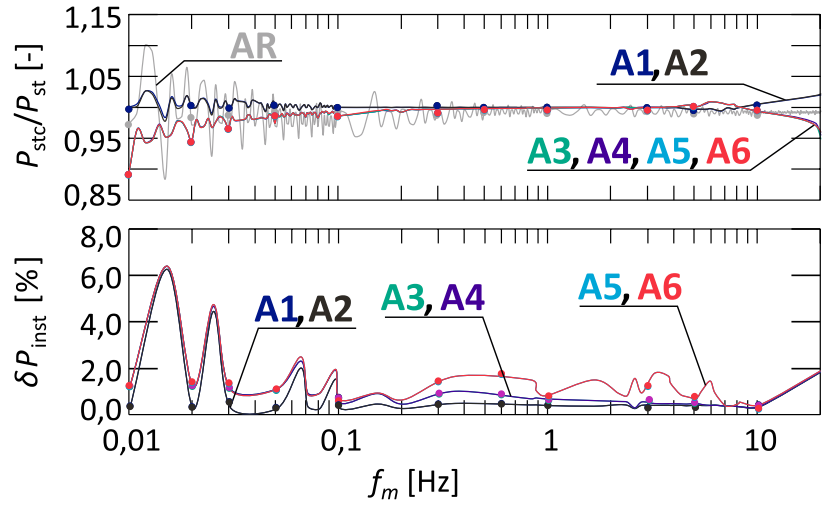

Fig. $8 P_{\text {stc }} / P_{\text {st }}=\mathrm{f}\left(f_{m}\right)$ (top) and $\delta P_{\text {inst }}=\mathrm{f}\left(f_{m}\right)$ (bottom) characteristic for $P_{\mathrm{st}}=1.2=$ const

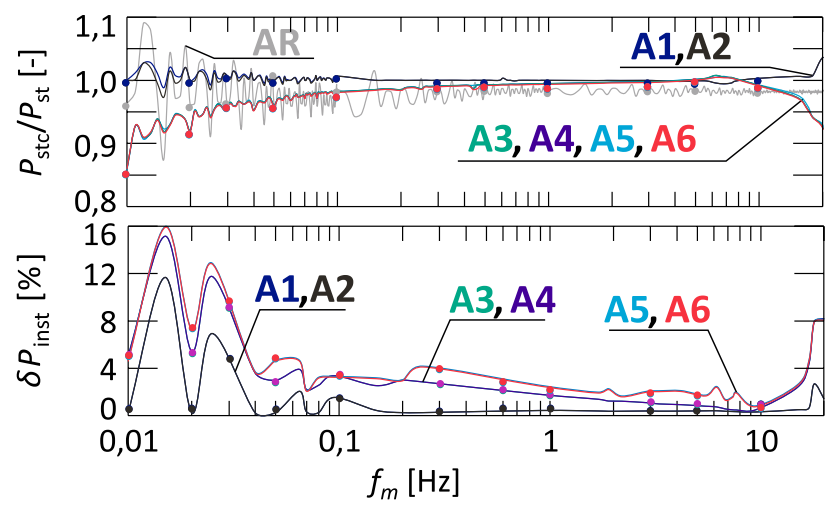

Fig. $9 P_{\text {stc }} / P_{\text {st }}=\mathrm{f}\left(f_{m}\right)$ (top) and $\delta P_{\text {inst }}=\mathrm{f}\left(f_{m}\right)$ (bottom) characteristic for $P_{\mathrm{st}}=3=$ const

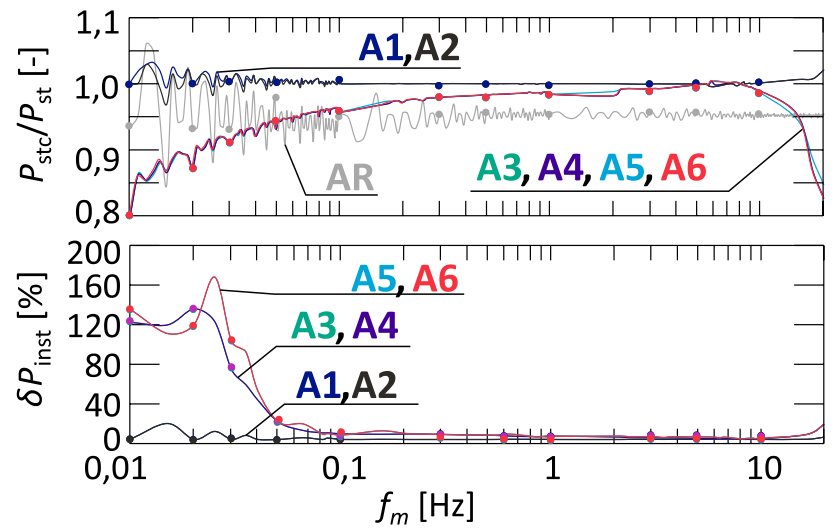

Fig. $10 P_{\text {stc }} / P_{\text {st }}=\mathrm{f}\left(f_{m}\right)$ (top) and $\delta P_{\text {inst }}=\mathrm{f}\left(f_{m}\right)$ (bottom) characteristic for $P_{\mathrm{st}}=5=$ const

include range of the obnoxious flicker. For each case, the $P_{s t c}$ and $P_{\text {instc }}$ were determined by supplying the AM modulated voltage with using recreated voltage envelope by the considered algorithms to the IEC flickermeter. Using $P_{s t c}$, the

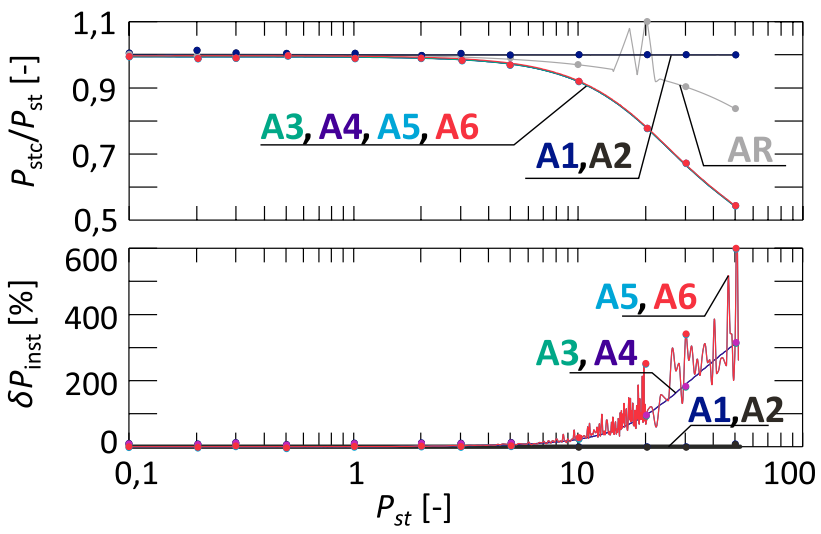

Fig. $11 P_{\text {stc }} / P_{\text {st }}=\mathrm{f}\left(P_{\text {st }}\right)$ (top) and $\delta P_{\text {inst }}=\mathrm{f}\left(P_{\text {st }}\right)$ (bottom) characteristic for $f_{m}=0.2 \mathrm{~Hz}=$ const

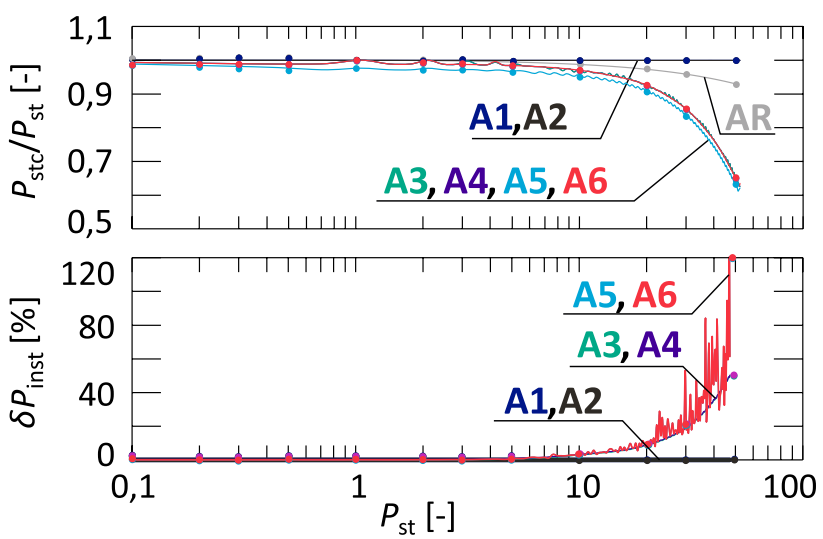

Fig. $12 P_{\text {stc }} / P_{\text {st }}=\mathrm{f}\left(P_{\text {st }}\right)$ (top) and $\delta P_{\text {inst }}=\mathrm{f}\left(P_{\text {st }}\right)$ (bottom) characteristic for $f_{m}=10 \mathrm{~Hz}=$ const

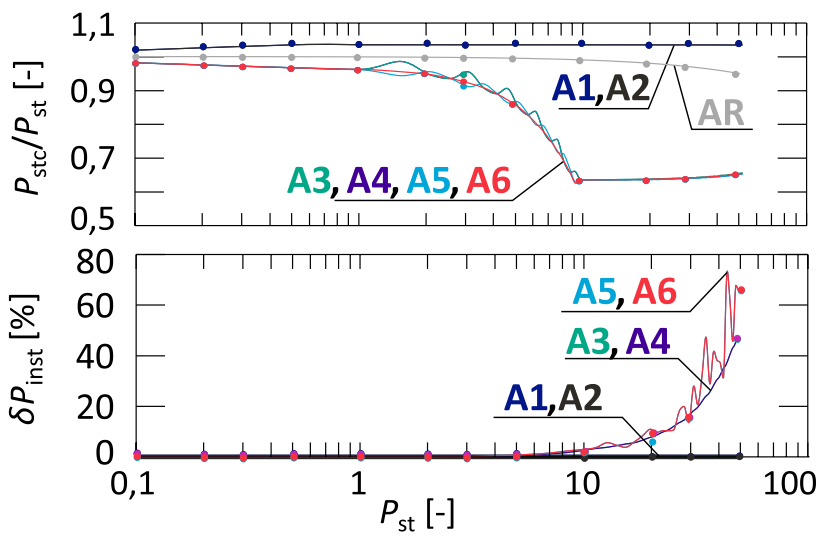

Fig. $13 P_{\text {stc }} / P_{\text {st }}=\mathrm{f}\left(P_{\text {st }}\right)$ (top) and $\delta P_{\text {inst }}=\mathrm{f}\left(P_{\text {st }}\right)$ (bottom) characteristic for $f_{m}=20 \mathrm{~Hz}=$ const

characteristics for normalized value of the indicator $P_{s t c} / P_{s t}$ were determined, which should always be equal to 1 in the case of the ideal operation of the algorithm. Using $P_{\text {instc }}$, the 


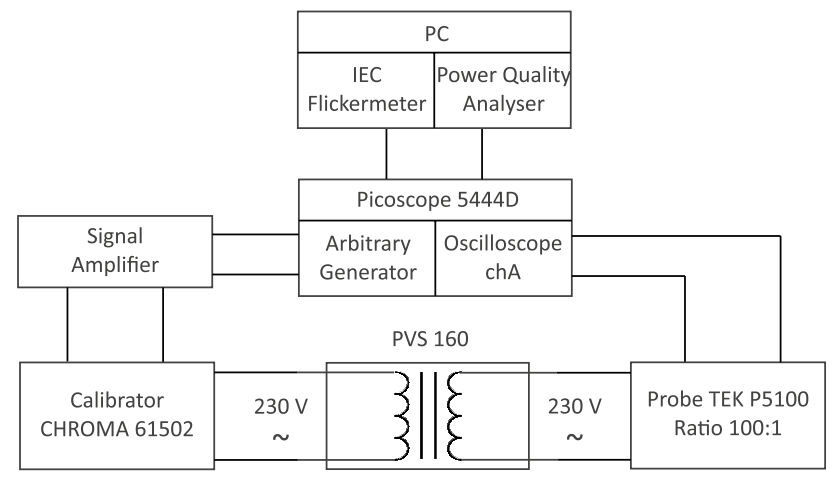

Fig. 14 The block diagram of the measuring system

characteristics of the average instantaneous flicker recovery error $\delta P_{\text {inst }}$ were determined according to the dependence:

$\delta P_{\text {inst }}=\frac{1}{N} \sum_{n=1}^{N} \frac{\left|P_{\text {inst }}(n)-P_{\text {instc }}(n)\right|}{P_{\text {inst }}(n)} \cdot 100 \%$,

where $N$ is the number of samples in the measurement interval. The error $\delta P_{\text {inst }}$ should always be equal to 0 in the case of the ideal operation of the algorithm. Because the graphs partially overlap, the line of individual waveforms cannot be observed.

Additionally, the correctness of the simulation was verified by laboratory studies, which were carried out for several selected points. These studies were carried out using: Picoscope 5444D generator/oscilloscope, CHROMA 61502 generator/amplifier, PQ BOX 100 power quality analyser. The block diagram of the measuring system is shown in Fig. 14. The results of experimental studies also were normalized to the $P_{s t c} / P_{\text {st }}$ and $\delta P_{\text {inst }}$, and were marked with "dots" on the individual characteristics.

In the considered cases, the characteristics have been limited to a modulation signal frequency $f_{m}$ equal to $20 \mathrm{~Hz}$, because the accurate of the algorithms for higher $f_{m}$ rapidly decreases, as shown in Fig. 15. This phenomenon results from the voltage indices calculating based on the rms value of voltage, determined every half-period, so some information for high-frequency modulation is lost [25]. However, the most disturbing loads change their operating state with a frequency less than $20 \mathrm{~Hz}$, except chaotic loads (e.g., arc furnaces) and power electronic devices.

Based on the characteristics shown in Figs. 3, 4, 5, 6, 7, 8, $9,10,11,12$ and 13 , when the frequency of the modulating signal $f_{m}$ is less than $20 \mathrm{~Hz}$, then the algorithms $\mathrm{A} 1$ and $\mathrm{A} 2$ allow the best recreation of the voltage envelope, because for the pre-set signal and recreated signal was obtained a comparable short-term flicker indicator $P_{s t}$. In the worst research conditions, the error of recreation for these methods is no more than $5 \%$, and in the range of typical voltage

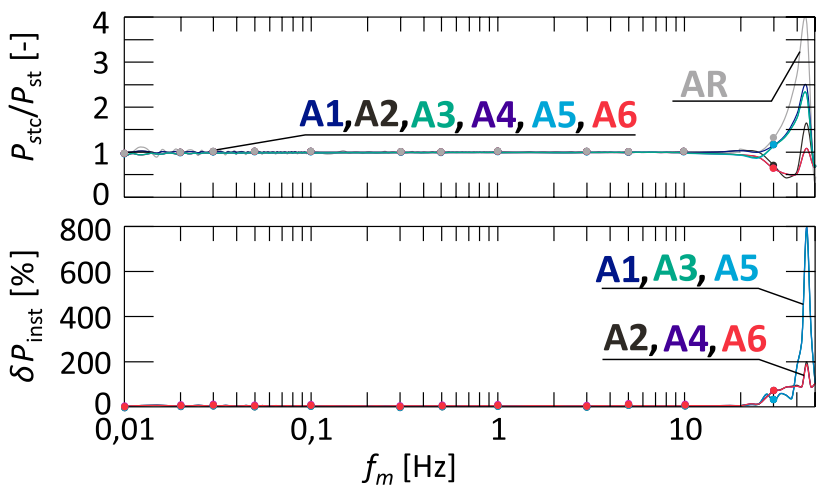

Fig. $15 P_{\text {stc }} / P_{\text {st }}=\mathrm{f}\left(f_{m}\right)$ (top) and $\delta P_{\text {inst }}=\mathrm{f}\left(f_{m}\right)$ (bottom) characteristic, which shows a rapid increase error value for $f_{m}$ greater than $20 \mathrm{~Hz}$

changes is no more than $0.01 \%$ (based on the characteristics of $\left.P_{s t c} / P_{s t}=\mathrm{f}\left(f_{m}\right)\right)$. The algorithms A1 and A2 allow more accurate recreation of the voltage envelope, compared to other literature solutions [15-19], including the algorithm AR.

Significantly worse results were obtained with other algorithms, i.e., A3, A4, A5, A6. In the case of these solutions, an increase in the modulation depth is resulted in a decrease in the accuracy of the obtained result. With a constant modulation depth, along with the increase of the frequency $f_{m}$, the accuracy of the obtained result increases up to a certain limit frequency, the exceeding of which causes a rapid decrease in the quality of the recreation of the voltage envelope. In addition, the obtained $P_{s t c}$ values for these algorithms were always smaller than the measured $P_{s t}$ values. The error tendency of voltage variation recreation results from the adopted assumptions, because the increase in the depth of modulation or frequency $f_{m}$ results in the change of the shape of recreated voltage envelope from the trapezoidal to the triangular. In turn, it results from [24] that the voltage modulated by a triangular signal causes the significant lower "obnoxious" flicker than modulation of the rectangular or trapezoidal signal. Therefore, to enable correct operation of these algorithms, it is important to measure the real speed of voltage changes. Furthermore, for the algorithms A4 and A6, it is also important to determination the standard deviation of the measured speed of voltage changes $S R$. The idea of these algorithms is based on real cases in which the change of the rms value of voltage is not always step change, e.g., when large motors are equipped with a softstart system. Considering the results of the simulation, it can be concluded that the quality of solutions obtained using the algorithms A3, A4, A5, A6 generates smaller errors than the existing literature solutions (error less than 5\%), but only for small values of modulation depth in the narrow range of the modulation signal frequency $f_{m}$. 


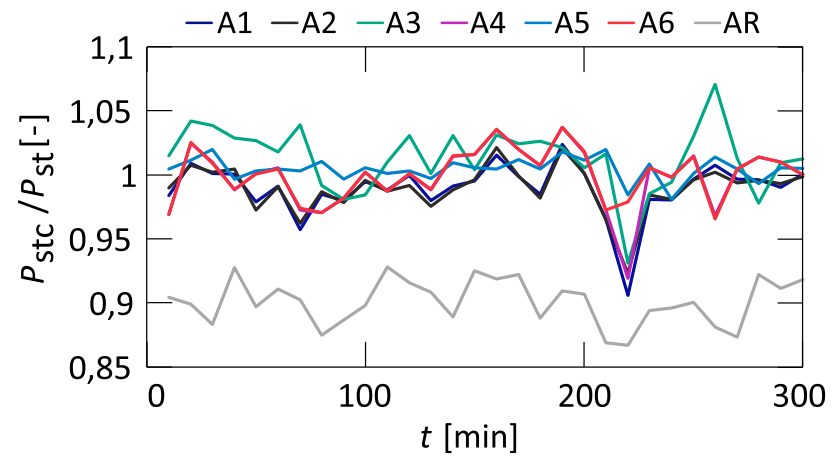

Fig. 16 Exemplary $P_{\mathrm{stc}} / P_{\mathrm{st}}=\mathrm{f}(t)$ characteristic obtained for the tested supply circuit of sewage pumping stations

Because the research aims to solve a practical problem, the quality of the presented algorithms was also verified based on real measurements from the sewage pumping station power circuit, where voltage fluctuations and data required to implement presented algorithms were monitored over a week. For the algorithms used, the error is usually no greater than $5 \%$. The smallest error was achieved using the probabilistic algorithm A5, which considering: the random operation of voltage fluctuations sources, and different speeds of voltage changes $S R$. The recreation of the voltage envelope based on voltage fluctuation indices allow estimation: the time interval in which the disturbing load operated; the level of disturbances it generates; and the frequency of its operation $[26,27]$. The exemplary results obtained for the 5-h interval are shown in Fig. 16.

\section{Conclusion}

In the article, the innovative algorithms allowing recreation of voltage variation (the voltage envelope) using parameters measured in the power grid have been presented. The correctness of the operation of individual algorithms has been verified based on the indicator of short-term flicker $P_{s t}$ obtained from measurements.

The simulation studies show that the algorithms A1 and A2 have the best properties. Both of them achieved much better accuracy than other algorithms available in the literature, because the error was not greater than $5 \%$ (based on the characteristics of $\left.P_{s t c} / P_{s t}=\mathrm{f}\left(f_{m}\right)\right)$. The remaining algorithms achieved satisfactory accuracy only at a low modulation depth in the narrow range of the modulation signal frequency. The inaccuracy of these algorithms is the result of the lack of information about the real average speed of voltage changes and its standard deviation, which is resulted in distortion of changes shape in the rms value of voltage at time. Obtaining information on the speed of voltage changes
$S R$ in the real power grid would make the algorithms A3, A4, A5, A6 more useful.

In the case of real circuit analysis, the recreation error for all algorithms was not greater than 5\% usually, and the smallest error values were obtained for the probabilistic algorithm A5. The inaccuracy for considered algorithms is resulted from the accepted limit value of the speed of voltage changes $S R$, for which voltage changes are classified as voltage fluctuations, because the voltage fluctuation indices used to recreation the voltage envelope do not include slow voltage changes, which can cause obnoxious flicker. However, an example of the application of individual algorithms on the real object, which is the supply circuit of sewage pumping stations, confirms the usability and accuracy of the proposed algorithms.

It is worth noting, that in laboratory studies (A1, A2) the best accuracy was obtained using a different algorithm than in the practical situation (A5). In practice, the speed of voltage changes $S R$ is not constant. In addition, voltage changes are caused by random operation of the source of disturbances, which does not always have to result in rapid changes in the rms value of voltage in the power grid. Probabilistic operation of the algorithm A5 considers these situations. Hence, algorithm A5 has obtained better accuracy in a real case. The recreating of these conditions in laboratory studies is a difficult task. Therefore in practice, modelled voltage fluctuations in laboratory studies were deterministic signals with constant speed of voltage changes $S R$. Therefore, the smallest errors in laboratory studies were obtained for the algorithms A1 and A2 with a constant speed of voltage change $S R$.

For recreated voltage envelopes using the proposed algorithms, higher errors were occurred with the estimation of instantaneous flicker $P_{\text {inst }}$ than with the estimation of $P_{s t}$, because the coding of voltage variation to voltage fluctuation indices is lossy coding. During coding, information about the shape and time of occurrence of voltage changes in the discrimination period is lost.

Although the accuracy of algorithms recreating voltage changes in relation to other algorithms available in the literature has been improved, it is still limited to voltage fluctuations whose source does not change its operating state with a frequency greater than $20 \mathrm{~Hz}$. Improvement of individual algorithms that would allow the research on all sources of voltage fluctuations is further studies of the authors, e.g., creation of a new method for estimating a modulating signal with a higher frequency than the carrier [28]; or creating a wireless measuring and recording device enabling measurement of the average value and standard deviation of the speed of voltage changes [29]. It is expected that the obtained results would allow for automatic identification and analysis of voltage fluctuation sources in the power grid with 
radial topology, to remove the disturbance that they emit [30].

Open Access This article is licensed under a Creative Commons Attribution 4.0 International License, which permits use, sharing, adaptation, distribution and reproduction in any medium or format, as long as you give appropriate credit to the original author(s) and the source, provide a link to the Creative Commons licence, and indicate if changes were made. The images or other third party material in this article are included in the article's Creative Commons licence, unless indicated otherwise in a credit line to the material. If material is not included in the article's Creative Commons licence and your intended use is not permitted by statutory regulation or exceeds the permitted use, you will need to obtain permission directly from the copyright holder. To view a copy of this licence, visit http://creativecommons.org/licenses/by/4.0/.

\section{References}

1. EN 50160:2010 Voltage characteristics of electricity supplied by public electricity networks

2. IEV number 604-01-19, Voltage fluctuation. http://std.iec.ch/iec60 050. Accessed 01 Oct 2019

3. Bayo AH (2008) Voltage fluctuations and flicker. In: Baggini A (ed) Handbook of power quality. Wiley, Hoboken, pp 135-163

4. Dugan RC et al (2003) Electrical power systems quality. MegrawHill, New York

5. Bollen MHJ (2003) What is power quality? Electric Power Syst Res 66(1):5-14

6. Simoes MC, Deckmann SM (2002) Flicker propagation and attenuation. In: Proceedings of the 10th international conference on harmonics and quality of power, vol 2, Rio de Janeiro, pp 644-648

7. Wiczynski G (2010) Study on measures of voltage fluctuation in electrical networks, habilitation dissertation. Poznan University of Technology, Poznan

8. Vega V et al (2008) Selecting wavelet function for detection power quality disturbances. In: 2008 IEEE/PES transmission and distribution conference and exposition: Latin America, pp 1-4

9. Garcia VV, Gualdrón CAD, Plata GO (2007) Obtaining patterns for classification of power quality disturbances using biorthogonal wavelets, rms values and support vector machines. In: Proceedings of 9th international conference electrical power quality and utilization, Barcelona, pp 1-6

10. Abdullah ARB, Sha'ameri AZB, Jidin AB (2010) Classification of power quality signals using smooth-windowed Wigner-Ville distribution. In: International conference on electrical machines and systems, Incheon, pp 1981-1985

11. Chen Q, Jia X, Zhao Ch (2009) Analysis on measuring performance of three flicker detecting methods. In: Proceedings of the IEEE PES 2009 general meeting, Calgary, pp 1-7

12. Wetula A, Bien A (2014) New measures of power-grid voltage variation: power delivery. IEEE Trans Power Deliv 29(3):1020-1027

13. Al-Hasawi WM, El-Naggar KM (2004) A genetic based algorithm for voltage flicker measurement. Electr Power Energy Syst 26:593-596

14. Marei MI, Shatshat R (2007) Fast envelope estimation technique for monitoring voltage fluctuations. J Electr Eng Technol 2(4):445-451

15. Michalski M (2018) Introductory results of tests of algorithms for recreation of voltage variation with voltage fluctuation indices. In: Proceedings of the 18th international conference on harmonics and quality of power, Ljubljana, pp. 1-5
16. Michalski M, Wiczynski G (2016) Determination of the parameters of voltage variation with voltage fluctuation indices. In: Proceedings of the 17th international conference on harmonics and quality of power, Belo Horizonte, pp 450-455

17. Michalski M, Wiczynski G (2018) An example of converting voltage fluctuation indices into Pst indicator. In: Proceedings of the 18th international conference on harmonics and quality of power, Ljubljana, pp 1-5

18. Wiczynski G (2017) Estimation of Pst indicator values on the basis of voltage fluctuation indices. IEEE Trans Instrum Meas 66(8):2046-2055

19. Michalski M (2017) Regeneration of power grids voltage variation with voltage fluctuation indices. Acad J Electr Eng 90:69-78

20. Kuwalek P (2019) The application of kernel density estimation for aided the process of locating sources of voltage fluctuations. Przeglad Elektrotechniczny 95(8):70-74

21. http://www.spe-energo.com/index.htm. Accessed 5 Dec 2018, 10:20

22. IEC Std. 61000-4-15:2003 Flickermeter-functional and design specifications

23. https://www.powerstandards.com/tutorials/what-is-flicker/. Accessed 3 Dec 2018, 09:11

24. Wiczynski G (2008) Analysis of voltage fluctuations in power networks. IEEE Trans Instrum Meas 57(11):2655-2664

25. Wiczynski G (2012) Comparison of voltage fluctuation measures. Poznan Univ Technol Acad J Electr Eng 72(2012): 9-16

26. Onal Y, Gerek ON, Ece DG (2016) Empirical mode decomposition application for short-term flicker severity. Turk J Electr Eng Comput Sci 24(2016):499-509

27. Xiaojing C, Kaicheng L, Qingxu M, Delong C, Yi L (2017) Detection of power quality disturbances using empirical wavelet transform and hilbert transform. J Electr Electron Eng 5(5):192-197

28. Kuwalek P (2019) AM modulation signal estimation allowing further research on sources of voltage fluctuations. IEEE Trans Ind Electron. https://doi.org/10.1109/tie.2019.2935978 (in press)

29. Jesko W, Kuwalek P (2019) The prototype of a wireless measurement card. In: ITM Web of Conference, vol 28, no 01045

30. Yan G et al (2017) Research on the mechanism of neutral-point voltage fluctuation and capacitor voltage balancing control strategy of three-phase three-level t-type inverter. J Electr Eng Technol 12(6):2227-2236

Publisher's Note Springer Nature remains neutral with regard to jurisdictional claims in published maps and institutional affiliations.

Piotr Kuwalek He received the M.Sc. Eng. degree in electrical engineering and B.Sc. degree in mathematics from the Poznan University of Technology, Poznan, Poland, in 2018. He is currently a Ph.D. student at the Faculty of Electrical Engineering at Poznan University of Technology. His current research interests include power quality evaluation and signal processing.

Waldemar Jesko He received the M.Sc. Eng. degree in electrical engineering from the Poznan University of Technology, Poznan, Poland, in 2018. He is currently a Ph.D. student at the Faculty of Computer Science at Poznan University of Technology. His current research interests include neural networks, speech recognition and speech signal processing. 